\title{
Graph Voronoi Regions for Interfacing Planar Graphs
}

\author{
Thomas Kämpke and Matthias Strobel \\ Forschungsinstitut für anwendungsorientierte Wissensverarbeitung FAW \\ Helmholtzstr. 16, 89081 Ulm, Germany \\ $\{$ kaempke,mstrobel\}@faw.uni-ulm.de
}

\begin{abstract}
Commanding motion is supported by a touch screen interface. Human input demonstrating trajectories by a sequence of points may be incomplete, distorted etc. Theses e ects are compensated by a transformation of vertex sequences of a regular grid into paths of a planar graph which codes feasible motions. The transformation is based on alteration operations including re-routings and on a so-called graph Voronoi regions which partition the plane according to proximity to vertices and edges.
\end{abstract}

Keywords: graph Voronoi region, grid graph, touch screen.

\section{Introduction}

Touch screen specifications of routes in a graph are investigated for the Euclidean space. A graph is therefore overlayed with a regular grid. The interplay between the graph and the grid gives rise to a variety of questions such as how to transform a sequence of grid points into a (meaningful) path in the graph. This task is similar to raster vector conversion with the difference that "vectors" cannot be chosen arbitrarily here but have to be taken from the graph. There is no true or ultimate transformation here since the intended path may adhere to ergonomical, aesthetic, or other criteria. Subsequent solutions should hence be considered as elements that may be combined in different manners.

The motivation for this problem stems from non-keyboard man-machine interfaces. Dynamic pointing operations typically serve for moving a scroll bar or an icon and for obtaining artistic effects from drawing with digital ink [2, p. 13]. Here, dynamic pointing relates to visible structures that restrict real motion in analogy to "streets". Applications of the approach include methods for input to spatial planning systems like navigation systems and techniques for commanding mobile systems by allowing explicit human guidance.

The difficulty of grid to graph transformations stems from the regular neighbourhoods of the grid and the irregular neighbourhoods of the graph being indepen- 
dent of each other. The transformations operate on two levels where the lower level utilizes geometric concepts while the upper level operates by production rules for regular expressions on mixed sequences of vertices and edges. So-called graph Voronoi regions will account for proximity towards vertices and edges.

\section{Graphs, Grids, and Their Relation}

Undirected graphs $G=(V, E)$ with vertex set $V$ and edge set $E$ are assumed to be simple meaning that there is at most one edge between any two vertices and no edge connects a vertex with itself (no loops). Each edge $e$ is labeled by a non-negative length $c(e)=c_{e}$. A path is a vertex sequence with successive vertices being adjacent. As the graph is simple, successive vertices of a path are distinct but revisiting vertices is feasible within a path. The cost of a path $P(v, w)=\left(v=v_{1}, \ldots, v_{s}=w\right)$ from $v$ to $w$ with $\left\{v_{1}, v_{2}\right\}, \ldots,\left\{v_{s-1}, v_{s}\right\} \in E$ is $c(P(v, w)):=\sum_{i=1}^{s-1} c\left(v_{i}, v_{i+1}\right)$. A shortest path from $v$ to $w$ is denoted $P_{0}(v, w)$. The degenerate case $P_{0}(v, v)$ is the single vertex $v$.

All graphs are planar, connected, and the length of a vertex sequence that need not be a path is considered later. The length of such a sequence is defined as sum of labels of successive vertices with cost assignment $d(v, u):=c(v, u)$ for $\{v, u\} \in E$ and $d(v, u):=c\left(P_{0}(v, u)\right)$ for $\{v, u\} \notin E$.

Actual point sets connecting vertices are denoted by $\operatorname{cur}\left(v_{1}, v_{2}\right)$. Edges and curves are symmetric in the sense that $e=\left\{v_{1}, v_{2}\right\}=\left\{v_{2}, v_{1}\right\}$ and $\operatorname{cur}\left(v_{1}, v_{2}\right)=$ $\operatorname{cur}\left(v_{2}, v_{1}\right)$. Curves may have several intersection points but no common sections.

Grids are unbounded and consist of equidistant horizontal and vertical lines. Each grid point has eight neighbours which are reachable by moving along lines and diagonals until the next point. The neighbourhood set of grid point $p$ is $N(p)$ and the extended neighbourhood includes the grid point itself, i.e. $N^{\prime}(p)=N(p) \cup\{p\}$.

A vertex with smallest Euclidean distance towards a grid point $p$ is $v(p)=$ $\operatorname{argmin}_{v \in V}\|v-p\|_{2}$ and a grid point with smallest Euclidean distance towards graph vertex $v$ is $p(v)=\operatorname{argmin}_{p \in P}\|p-v\|_{2}$. The grid is assumed to be finer than the graph meaning that distinct graph vertices lie apart by at least the grid width. Thus, distinct graph vertices have distinct closest grid points. The Voronoi region of a vertex is the set of all points with smaller distance to that vertex than to any other vertex, $V(v)=\{x \mid\|x-v\| \leq\|x-w\| \forall w \in V-\{v\}\}$ with $v$ being the center of the Voronoi region. When clear from the context, Voronoi regions will consist only of the grid points contained in the proper Voronoi regions.

\section{Sequence Transformations}

A grid point sequence $\bar{p}=\left(p^{(1)}, \ldots, p^{(N)}\right)$ induces the sequence of closest graph vertices $\bar{v}(\bar{p})=\left(v\left(p^{(1)}\right), \ldots, v\left(p^{(N)}\right)\right)$. The grid point sequence is connected if each grid point is an extended neighbour of its predecessor. Even a connected 

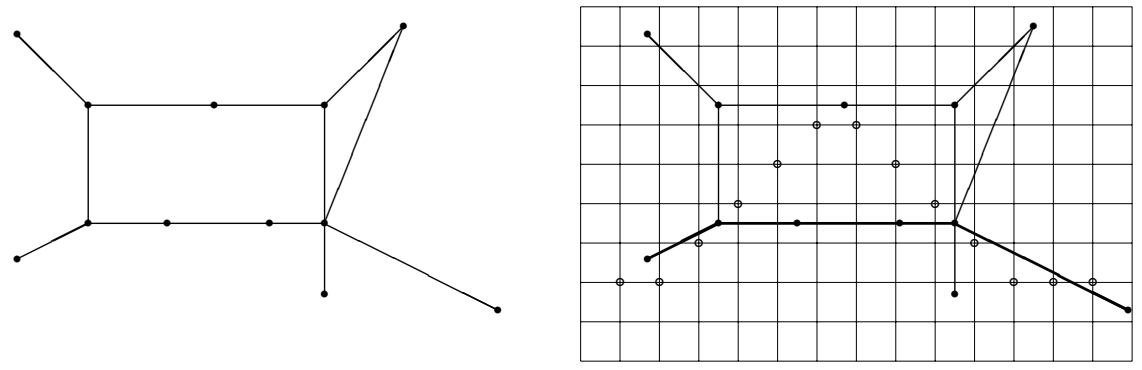

Figure 1: When specifying the grid point sequence (white dots) for a path (bold edges), the grid and the Voronoi regions are invisible (left).

grid point sequence need not induce a path. This property is addressed by forming traces which indicate the changes in vertex sequences. The trace of sequence $\left(v^{(1)}, \ldots, v^{(M)}\right)$ with $v^{\left(j_{1}\right)}=v^{(1)}=v^{(2)}=\ldots=v^{\left(j_{2}-1\right)} \neq v^{\left(j_{2}\right)} \ldots \neq$ $v^{\left(j_{M}\right)}=v^{(M)}$ is the subsequence $\operatorname{tr}\left(v^{(1)}, \ldots, v^{(M)}\right)=\left(v^{\left(j_{1}\right)}, \ldots, v^{\left(j_{M}\right)}\right)$. An example is $\operatorname{tr}\left(v_{4}, v_{4}, v_{3}, v_{4}, v_{5}, v_{5}, v_{4}, v_{7}\right)=\left(v_{4}, v_{3}, v_{4}, v_{5}, v_{4}, v_{7}\right)$. Whenever the trace is a path, this path is taken as the transform of the grid point sequence. In other cases, vertex insertions and deletions are required.

\subsection{Isolated Insertions and Deletions}

A vertex sequence which is not a path can be transformed into a path by vertex insertions between successive vertices with $\left\{v^{(i)}, v^{(i+1)}\right\} \notin E$. Vertex insertions will be obtained from shortest paths and they may adhere to additional constraints such as not using vertices from the present sequence or from other insertions. Decisions on allowing vertex repetitions ultimately appear to be possible only by convention rather than by purely geometrical criteria. Vertex deletion may serve as an alternative to insertions but this cannot be guaranteed to result in a path.

\subsection{Joint Insertions and Deletions}

Joint insertions and deletions also known as indels from string editing [3] adhere to connectivity. Therefore, a vertex of a sequence is understood to be isolated from the sequence, if the vertex is neither joined to its predecessor nor to its successor. A vertex that is not isolated from a sequence is connected to that sequence. A vertex $v^{(i)}$ is understood to be a single isolated vertex if $\left\{v^{(i-2)}, v^{(i-1)}\right\} \in$ $E,\left\{v^{(i-1)}, v^{(i)}\right\} \notin E,\left\{v^{(i)}, v^{(i+1)}\right\} \notin E$, and $\left\{v^{(i+1)}, v^{(i+2)}\right\} \in E$.

A vertex sequence receives insertions so that a single isolated vertex $v^{(i)}$ becomes connected if it lies on at least one shortest path from $v^{(i-1)}$ to $v^{(i+1)}$. Otherwise the vertex is deleted and again a shortest path is inserted from $v^{(i-1)}$ to $v^{(i+1)}$, compare figures 2 and 3 . 


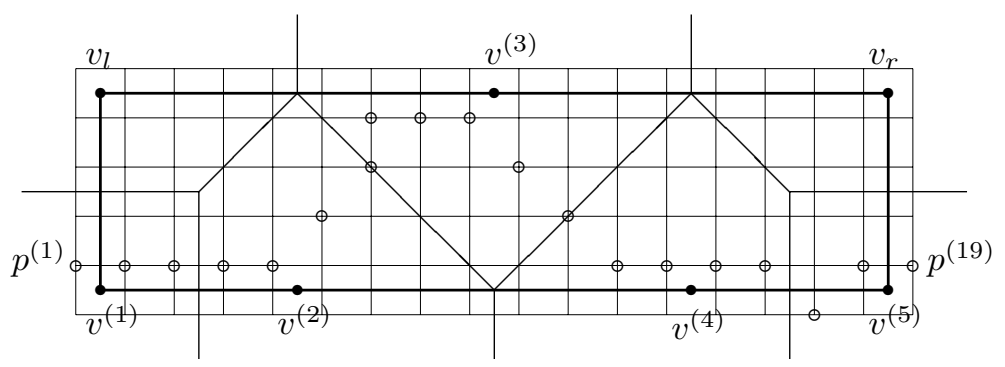

Figure 2: Graph with edges given by bold lines. Thin lines specify the boundaries of the Voronoi regions. Vertex $v^{(3)}$ is singly isolated in $\left(v^{(1)}, \ldots, v^{(5)}\right)$ which is induced by the connected sequence $\left(p^{(1)}, \ldots, p^{(19)}\right)$ indicated by white dots.

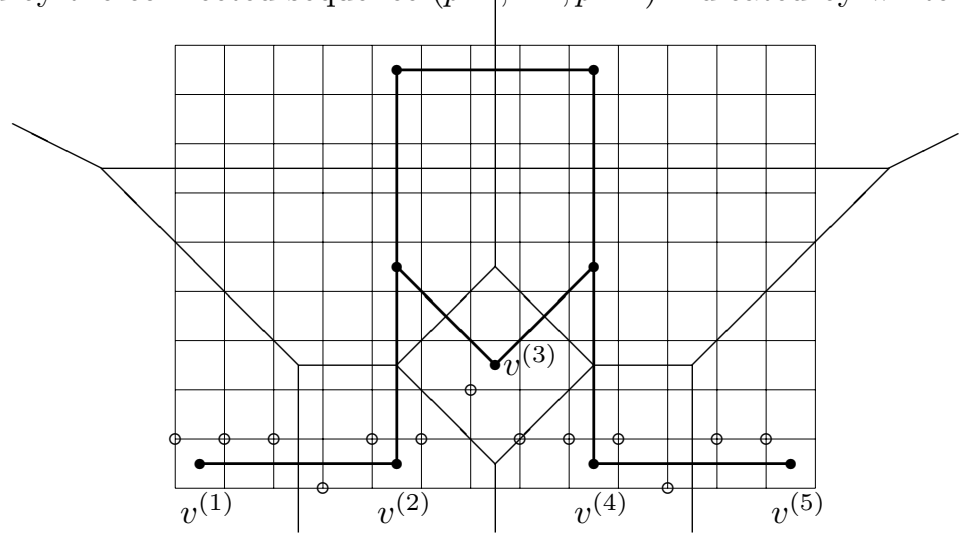

Figure 3: Vertex $v^{(3)}$ again is a singly isolated. As it is located on the unique shortest path from $v^{(2)}$ to $v^{(4)}$, it becomes connected to the vertex sequence.

\subsection{Graph Voronoi Regions for Planar Graphs}

Vertex proximity as expressed by Voronoi regions is not suitable for path specification. The reason is that closely following an edge which traverses a Voronoi region but is not incident with that region's center suggests an unintended vertex visit, compare figure 4. An appropriate partition of the plane is offered by forming certain Voronoi regions within Voronoi regions. These are based on the distance between a set $A$ and a point $x$ with respect to a Voronoi region:

$$
\operatorname{dist}_{V(v)}(x, A):= \begin{cases}\inf _{a \in A \cap V(v)}\|x-a\|, & \text { for } A \cap V(v) \neq \emptyset \\ \infty, & \text { for } A \cap V(v)=\emptyset .\end{cases}
$$

The graph Voronoi regions are established to express proximity to any graph element. Whenever a point from an ordinary Voronoi region is closest to that region's center or to an edge incident with the center, the point's assignment to the Voronoi region remains unchanged. Whenever a point from an ordinary 


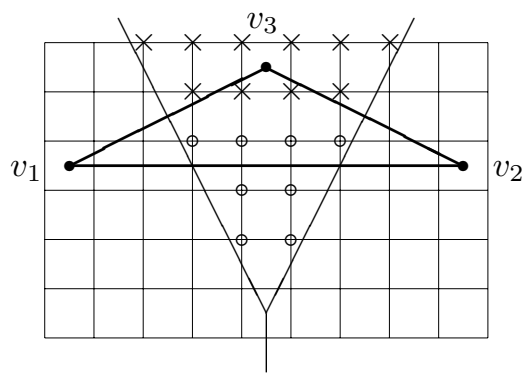

Figure 4: A connected grid point sequence following closely the edge $\left\{v_{1}, v_{2}\right\}$ leads to vertex $v_{3}$ being included in the induced vertex sequence. The mixed Voronoi region $V_{V\left(v_{3}\right)}\left(\operatorname{cur}\left(v_{1}, v_{2}\right)\right)$ contains all grid points marked by white dots. The remaining pure Voronoi region contains the grid points marked by crosses.

Voronoi region is closest to an edge that is not incident with the center of the Voronoi region, that edge receives a subset of the Voronoi region and the point under consideration is assigned to that subset. This results in the subsequent definitions of pure and mixed (graph) Voronoi regions.

$$
\begin{aligned}
& V_{V(v)}(v):=\{x \in V(v) \mid \exists \operatorname{cur}\left(v, v_{i}\right) \text { such that } \operatorname{dist}_{\mathbb{R}^{2}}\left(x, \operatorname{cur}\left(v, v_{i}\right)\right) \leq \\
& \operatorname{dist}_{\mathbb{R}^{2}}\left(x, \operatorname{cur}\left(v_{k}, v_{l}\right)\right), \\
&\left.\forall \operatorname{cur}\left(v_{k}, v_{l}\right) \text { with } v_{k}, v_{l} \in V-\{v\}\right\} \\
& V_{V(v)}\left(\operatorname{cur}\left(v_{i}, v_{j}\right)\right):=\{x \in V(v) \mid \operatorname{dist}_{\mathbb{R}^{2}}\left(x, \operatorname{cur}\left(v_{i}, v_{j}\right)\right) \leq \operatorname{dist}_{\mathbb{R}^{2}}\left(x, \operatorname{cur}\left(v_{k}, v_{l}\right)\right), \\
& \forall\left.\forall \operatorname{cur}\left(v_{k}, v_{l}\right) \text { with }\left\{v_{k}, v_{l}\right\} \neq\left\{v_{i}, v_{j}\right\}\right\} \text { for } \\
& v_{i}, v_{j} \in V-\{v\} .
\end{aligned}
$$

A pure Voronoi region coincides with the ordinary Voronoi region if and only if all its mixed Voronoi regions are empty. For graph Voronoi regions a curve traversing an ordinary Voronoi region without being incident with the center affects grid points of this region in the same way as a curve that passes by.

A grid point from a pure Voronoi region $V(v)$ or $V_{V(v)}(v)$ will induce vertex $v$ and a grid point from a mixed Voronoi region $V_{V(v)}\left(\operatorname{cur}\left(v_{i}, v_{j}\right)\right)$ will induce the edge $\left\{v_{i}, v_{j}\right\}$. Formally, for any $p \in P$

$$
\operatorname{ind}(p):= \begin{cases}v, & p \in V(v) \text { or } V_{V(v)}(v) \text { for some } v \\ \left\{v_{i}, v_{j}\right\}, & p \in V_{V(v)}\left(\operatorname{cur}\left(v_{i}, v_{j}\right)\right) \text { for some } v \text { and } v_{i}, v_{j} \in V-\{v\} .\end{cases}
$$

\subsection{From Mixed Sequences to Paths}

\subsubsection{Operations on Sequences}

A sequence of vertices and edges is called a mixed sequence. The mixed sequence induced by a grid point sequence $\bar{p}=\left(p^{(1)}, \ldots, p^{(N)}\right)$ is denoted by $\operatorname{ind}(\bar{p})=$ $\left.\operatorname{ind}\left(p^{(1)}\right), \ldots, \operatorname{ind}\left(p^{(N)}\right)\right)$. The trace of a mixed sequence is understood in analogy 
to the trace of a vertex sequence. Mixed sequences are transformed to vertex sequences according to a set $\mathcal{O}$ of operations. Their specification is based on strings such as $A[x], A[x, y]$ etc. which denote possibly empty strings like $A[x]=$ $x, x$ and $A[x, y]=y, y, x, y . A_{1}[x], A_{1}[x, y]$ etc. denote strings that consist of at least one of the bracketed terms. The vertex sequence resulting from no further operation of $\mathcal{O}$ being applicable is denoted by $\bar{v}(\cdot)$.

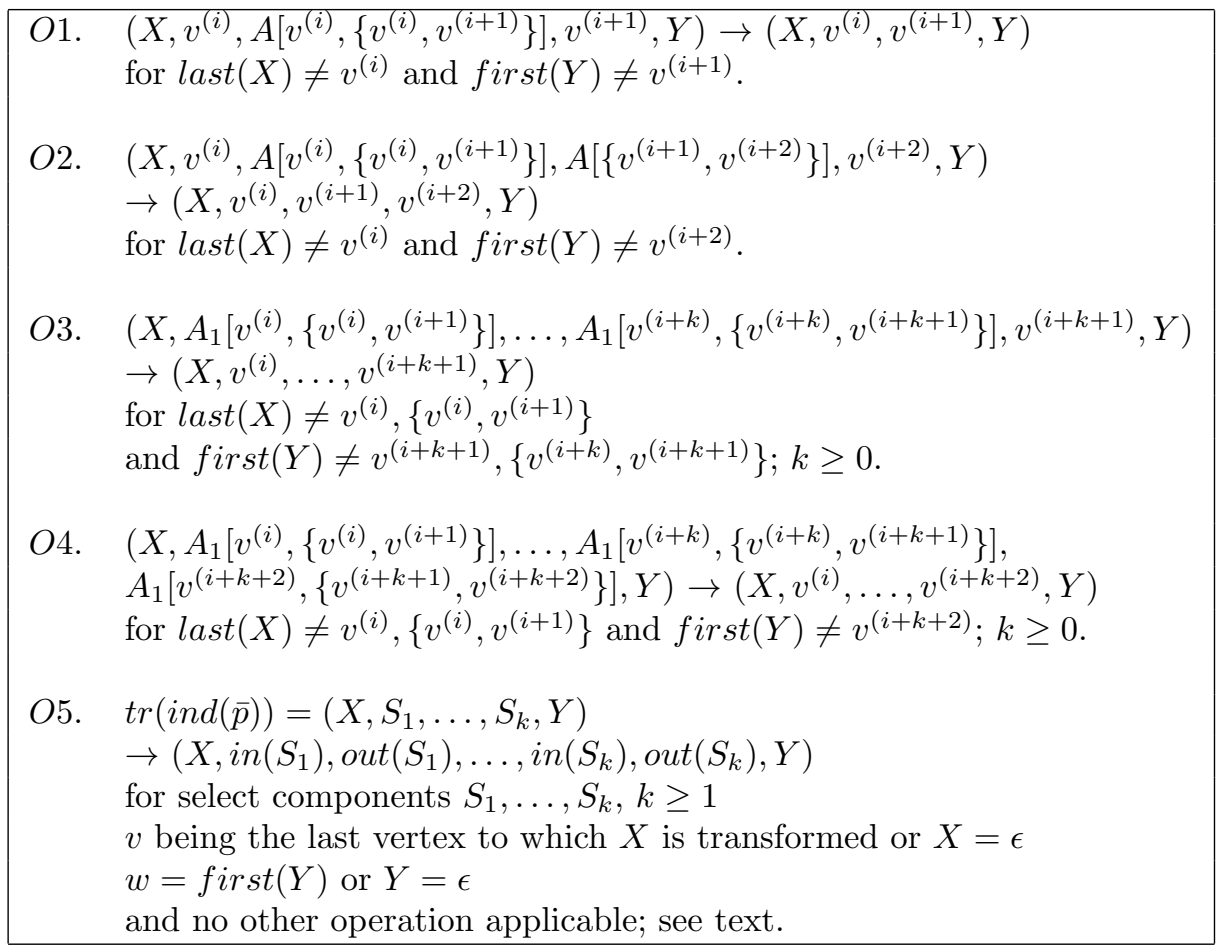

The prefix $X$ and the suffix $Y$ may be the empty string $\epsilon$. In case several successive edges neither share a vertex with their predecessor nor with their successor, operations $O 1$ - $O 4$ may not be applicable or may result in multiple sequence ambiguities. Such cases are resolved by select components. A select component of a mixed sequence is defined to be a $\subseteq$-maximal subsequence of successive vertices and edges such that it is either a single vertex, or a single edge, or applications of $O 1$ - $O 4$ lead to a unique vertex sequence.

Each select component has an entry vertex and an exit vertex which is unique in case the select component is a single vertex or leads to a unique vertex sequence. Otherwise, these vertices admit a twofold ambiguity. In the unique case, the entry vertex and the exit vertex may be identical as for a single vertex or a complete cycle. Unique entry and exit vertices are denoted by $v\left(i n, S_{i}\right)$ and $v\left(\right.$ out,$\left.S_{i}\right)$, the others are denoted by $v\left(\right.$ in $\left., S_{i}, 1\right), v\left(\right.$ in $\left., S_{i}, 2\right), v\left(\right.$ out $\left., S_{i}, 1\right)$, and $v\left(\right.$ out $\left., S_{i}, 2\right)$, where $v\left(\right.$ in $\left., S_{i}, 1\right)=v\left(\right.$ out $\left., S_{i}, 2\right)$ and $v\left(\right.$ in $\left., S_{i}, 2\right)=v\left(\right.$ out $\left., S_{i}, 1\right)$. Ambiguities are resolved by forming shortest paths as in figure 5 . All edges receive $d(\cdot, \cdot)$ labels with $d(v, \cdot)$ and $d(\cdot, w)$ becoming zero in case $X=\epsilon$ and $Y=\epsilon$ respectively. 


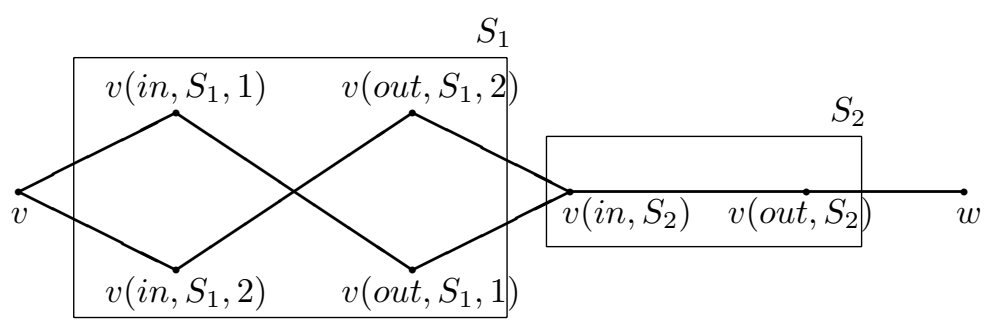

Figure 5: Substitution graph for shortest paths through $S_{1}$ and $S_{2}$ where $S_{1}$ has non-unique entry and exit vertices while $S_{2}$ has unique entry and exit vertices.

\subsubsection{Transformations}

Whenever a vertex sequence results from the operations of $\mathcal{O}$ applied to a mixed sequence, the vertex sequence has no immediate repetition. The transformation based on the extended definition of $\bar{v}$ formally is again given by

$$
\operatorname{Tr}(\bar{p}):= \begin{cases}\bar{v}(\operatorname{tr}(\operatorname{ind}(\bar{p}))), & \text { if } \bar{v}(\operatorname{tr}(\operatorname{ind}(\bar{p}))) \text { is a path in } G \\ \operatorname{void}, & \text { else. }\end{cases}
$$

Whenever the trace of an induced sequence alternates between two vertices such as $\left(v_{4}, v_{2}, v_{4}, v_{2}\right)$, no operation of $\mathcal{O}$ applies and thus the sequence is left unchanged by $\bar{v}(\cdot)$. It is thus possible to state deliberate vertex repetitions in paths by suitable grid point sequences.

\subsubsection{Complete Transformations}

In case $\operatorname{Tr}(\bar{p})$ is void, the vertex sequence $\bar{v}(\operatorname{tr}(i n d(\bar{p})))$ can be extended to a path by inserting shortest paths between any successive vertices that are not adjacent in $G$. A reasonable decision on inserting and deleting vertices can be based on the connectivity of the grid point sequence. If the grid point sequence is disconnected, deletions are forbidden. The reason is that a disconnection of the grid point sequence may result from deliberate jumps to sections of the graph that must be visited by the path. If the grid point sequence is connected, single isolated vertices will be deleted if they do not lie on a shortest connecting path in $G$, otherwise they will be connected. The complete procedure is as follows.

\section{A1}

1. Input $\bar{p}$ with $\operatorname{Tr}(\bar{p})=\bar{v}(\operatorname{tr}(\operatorname{ind}(\bar{p})))=\left(v^{(1)}, \ldots, v^{(M)}\right)=\bar{v}$.

2. If $\operatorname{Tr}(\bar{p})$ is a path, no operations are performed;

else if $\bar{p}$ is disconnected then any $v^{(i)}, v^{(i+1)}$ with $\left\{v^{(i)}, v^{(i+1)}\right\} \notin E$ are connected by $P_{0}\left(v^{(i)}, v^{(i+1)}\right)$ giving new path $\bar{v}$;

else insertions $P_{0}\left(v^{(i)}, v^{(i+1)}\right), P_{0}\left(v^{(i+1)}, v^{(i+2)}\right)$ are replaced by $P_{0}\left(v^{(i)}, v^{(i+2)}\right)$ if $v^{(i+1)}$ is single isolated vertex in original $\bar{v}$ giving new $\bar{v}$.

3. Output path $\bar{v}$. 
Whenever vertex repetitions in the final path are unintended they can be suppressed by best shortenings [5]. If connectivity of the grid point sequence is no criterion of the path construction, potential deletion of a single isolated vertex can still be considered meaningful giving the next algorithm.

\section{A2}

1. Input $\bar{p}$ with $\operatorname{Tr}(\bar{p})=\bar{v}(\operatorname{tr}(\operatorname{ind}(\bar{p})))=\left(v^{(1)}, \ldots, v^{(M)}\right)=\bar{v}$.

2. If $\operatorname{Tr}(\bar{p})$ is a path, no operations are performed;

else any $v^{(i)}, v^{(i+1)}$ with $\left\{v^{(i)}, v^{(i+1)}\right\} \notin E$ are connected by $P_{0}\left(v^{(i)}, v^{(i+1)}\right)$ giving new path $\bar{v}$, insertions $P_{0}\left(v^{(i)}, v^{(i+1)}\right)$ and $P_{0}\left(v^{(i+1)}, v^{(i+2)}\right)$ are replaced by $P_{0}\left(v^{(i)}, v^{(i+2)}\right)$ if $v^{(i+1)}$ is single isolated vertex in original $\bar{v}$.

3. Output path $\bar{v}$.

\section{Computational Issues}

The computation of graph Voronoi regions can be reduced to computing Voronoi regions of vertices and of a finite collection of line segments and then taking certain intersections of these regions. Both individual computations can be perforemd in $O(n \log n)$, see [9] for the latter, but these computations are concepturally complicated. They have even lead to approximations of Voronoi regions of a finite collection of line segments by angular bisector regions [1].

A simple approximation of graph Voronoi regions relies on the nearest vertex and the nearest edge for each grid point being computable in $O(n)$; planarity of the graph ensures that it has at most $3 n-6$ edges. Whenever the nearest line is incident with the nearest vertex, the grid point lies in the pure Voronoi region of that vertex. Otherwise, the grid point lies in the mixed Voronoi region of that line with respect to the nearest vertex. Computing the mixed induced sequence of a grid point sequence of legth $N$ then requires time $O(N n)$.

The previous approximations of graph Voronoi regions and transformations by algorithm A2 were implemented with input obtained from an elo 151R IntelliTouch 15-inch touch screen. Geometric data handling was organized within the LEDA system, and the algorithms were written in $\mathrm{C}++$.

Figures 6 through 9 show a planar graph and a sequence of about 200 grid points being transformed to a path. Edges are labeled by their Euclidean lengths. Figure 8 depicts approximations of the graph Voronoi regions. The "overshoot" (U-shaped section) of the grid point sequence in figure 7 in the center region of the graph is so large that vertex insertions occur. 


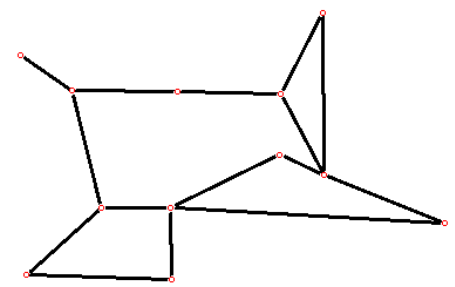

Figure 6: Planar graph with 12 vertices.

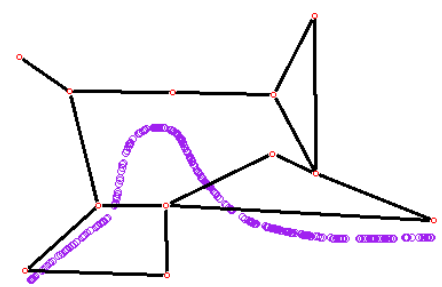

Figure 7: Graph with grid point sequence, grid omitted.

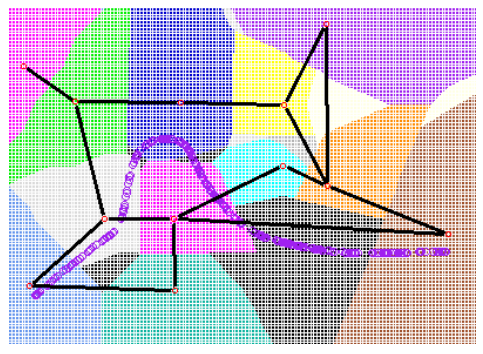

Figure 8: Graph, grid point sequence, and approximated graph Voronoi regions.

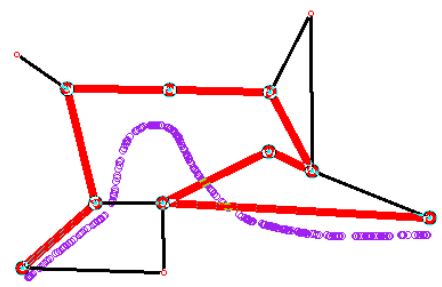

Figure 9: Graph with grid point sequence transformed to a path (bold edges). 


\section{References}

[1] Cloppet, F., Olivia, J.-M., Stamon, G., "Angular bisector network, a simplified generalized Voronoi diagram: applications to processing complex intersections in biomedical images", IEEE Transactions on Pattern Analysis and Machine Intelligence 22, 2000, p. 120-128.

[2] Cohen, P. et al., "Multimodal interaction for 2D and 3D environments", Computer Graphics and Applications, July/August 1999, p. 10-13.

[3] Gusfield, D., "Algorithms on strings, trees, and sequences: computer science and computational biology", Cambridge University Press, Cambridge, 1997.

[4] Hopcroft, J.E., Ullman, J., "Introduction to automata theory, languages and computation", Addison-Wesley, New York, 1979.

[5] Kämpke, T., "Interfacing graphs", Journal of Machine Graphics and Vision 9,2000 , p. 797-824.

[6] Leeuwen, J.v. (ed.), "Handbook of theoretical computer science: algorithms and complexity", vol. A, Elsevier, Amsterdam, 1990.

[7] O'Rourke, J., "Computational Gemetry in C", 2nd ed., Cambridge University Press, Cambridge, 1998.

[8] Sugihara, K., "Approximations of generalized Voronoi diagrams by ordinary Voronoi diagrams", Computer Vision and Graphic Image Processing 55, 1993, p. 522-531.

[9] Yap, C.K., " An $O(n * \log n)$ algorithm for Voronoi diagrams of a set of simple curve segments", Discrete and Computational Geometry 2, 1987, p. $365-393$. 